\section{Cell synchronization and dynamic G-banding of equine chromosomes by bromodeoxyurldine}

\section{L. Rlcher and A. Romagnano}

ABSTRACT: Both dynamic G-banding and cell synchronization produced by bromodeoxyuridine (BrdU), were applled to equine chromosomes. BrdU incorporated during the first half of the Sphase is taken up into the R-bands that are earty replicating. These bands, which have incorporated BrdU, cannot contract as usual and remain elongated; only the other regions of the chromosome, i.e., the G-bands, contract normally and are sharply defined. BrdU also can be used for cell synctronization. The addition of BrdU in a high concentration, 15 hours before harvest, and its removal 11 hours later, has two effects: Intthally the BrdU is incorporated during the first part of the S-phase and then It blocks the cells at midS-phase. Within the cell cycle, mid-S-phase appears to be the most vulnerable time to various blocking agents. To dlfferentlate the reglons of BrdU incorporation from those that have not been substituted, the fluorescence-photolysisGlemsa (FPG) tochnlque was applied as modlfted for horse chromosomes. This dynamic technlque, which produces many prometaphase and prophase chromosomes showing very sherp Gbands, is certain to entance the accuracy of cytogenetic analysis and aid In the standardizetion of equine chromosomes.

BROMODEOXYURIDINE (BrdU) has proven to be a useful tool for production of R-bands in horse chromosomes 5,6 . Indeed, R-bands are obtained when $\mathrm{BrdU}$ is added during the second half of the synthesis phase (late-S-phase) in the cell cycle. Furthermore, combined with a thymidine block, it can R-band prometaphasic horse chromosomes?. Conversely, if BrdU is added in the first half of the S-phase (early-Sphase), it will not only produce G-banding, but also, if present in a sufficient amount, will block the dividing cells at mid-S-phase resulting in cell synchronization'. Here we present our ad-

The authors are affiliated with the Departement d'Anatomie, Faculte de MEdecine, Universite de Montrtal, CP. 6128, Succ. A, Montrtal, Quebec, Canada H3C 3J7; and with the Centre de Recherche en Reproduction Animale, Faculté de MEdecine Vétérinaire, Université de Montréal, Montréal, Québec, Canada. Part of this work was presented at the 6th European Colloquium on Cytogenetics of Domestic Animals, Zurich, July 1984. The research was supported by the "Fonds F.C.A.C. pour l'aide et le soutien a la recherche", Quebec. The authors wish to thank Elise MEnard-Landry, Brigitte L'HermiteBeugnot, and Louise Laquerre for helpful assistance. Please address reprint requests to Dr. Richer. - 1985, American Genetic Association.

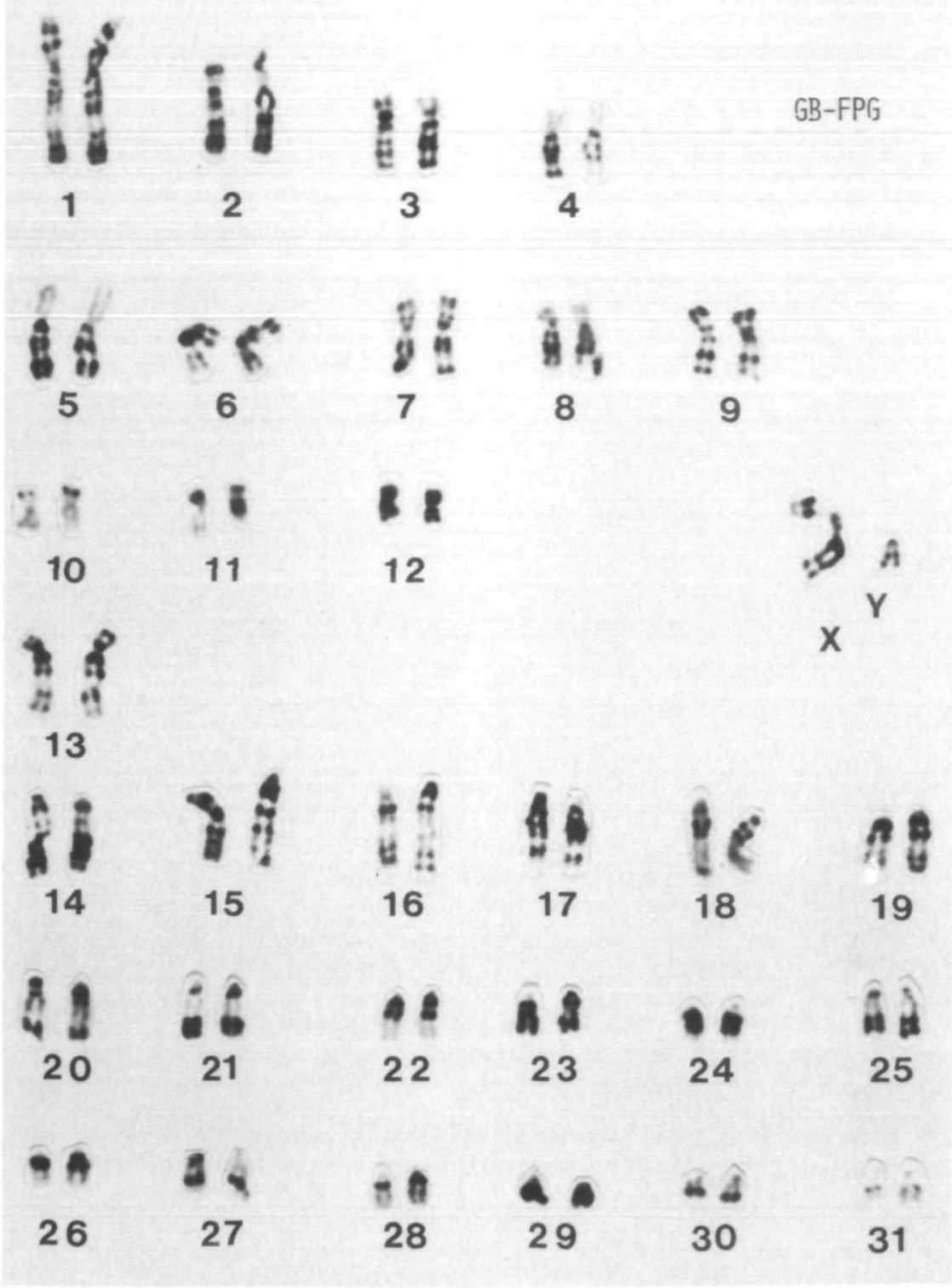

FIGURE 1 Prometaphase GB-FPG karyotype of a male domestic horse

aptation to equine chromosomes of this dynamic G-banding and cell-synchronization technique.

\section{Materials and Methods}

Peripheral blood was collected directly from the jugular vein of 10 horses into green stoppered Vacutainer tubes containing 143 USP units of sodium heparin (Becton Dickinson). Cultures were set up with $0.2 \mathrm{ml}$ of whole blood in $5 \mathrm{ml}$ of HB-103 medium (Hana Media) supplemented with $1 \mathrm{mM}$ glutamin (Flow), 2 percent Pokeweed mitogen (Gibco), and 0.01 $\mathrm{mg} / \mathrm{ml}$ of Garamycin (Schering). Following a 57 -hour incubation period at $37^{\circ} \mathrm{C}, 200 \mu \mathrm{g} / \mathrm{ml}$ of $\mathrm{BrdU}$ were added and 11 hours later cultures were rinsed twice with warm phosphate buffered saline, suspended in fresh medium containing $3 \mu \mathrm{g} / \mathrm{ml}$ of thymidine, and reincubated at $37^{\circ} \mathrm{C}$ for three to five hours.

At the end of this culture period the suspen- sion was centifuged and the cell button was then resuspended in $0.075 \mathrm{M} \mathrm{KCl}$ and reincubated for 20 minutes. After this hypotonic treatment the cells were fixed, first in methanol:chloroform:acetic acid (3:2:1) and then twice in methanol:acetic acid (3:1). This suspension was spread on cold $\left(0^{\circ} \mathrm{C}\right)$ slides and air dried at room temperature. The fluorescence-photolysis-Giemsa (FPG) method ${ }^{2,4}$, as previously described for horse chromosomes ${ }^{5,6}$ was used to visualize the G-banding thus produced. Preparations were analyzed and photographed under a light microscope.

G-bands produced by trypsin and stained with Giemsa (GTG-bands) were prepared, on other slides, according to the technique of Seabright $^{8}$.

\section{Results and Discussion}

Although the percentage of dividing cells is slightly decreased as compared to the standard 

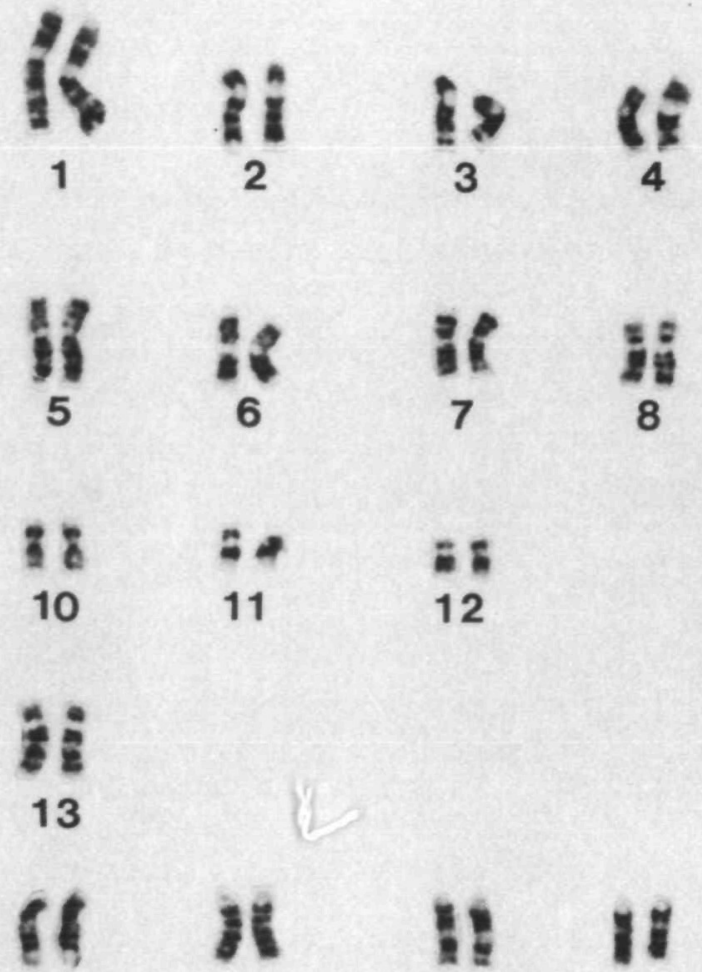

11

12
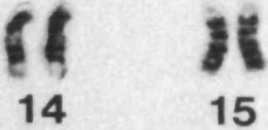

15

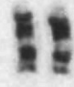

16

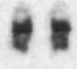

22

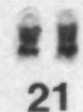

20

21

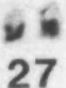

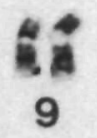

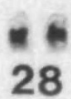

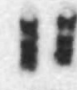

17
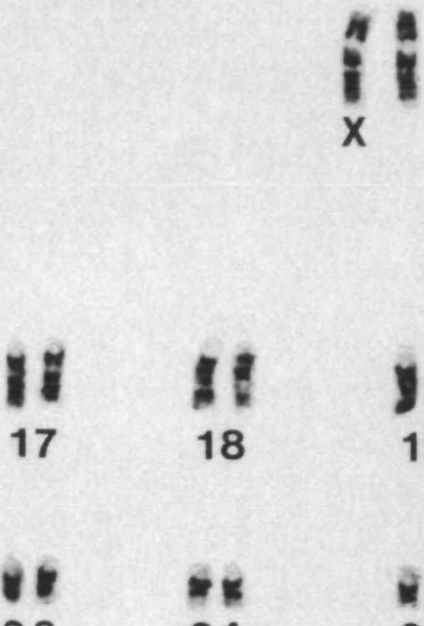

23

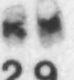

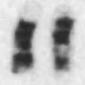

18

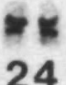

24

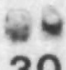

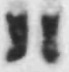

19

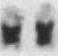

25

$-$

31
FIGURE 2 Early metaphase GTG karyotype of a female domestic horse.

whole blood technique, a sufficient number is obtained. If only 3 to 5 hours elapse between lifting the cell block and harvesting, the majority of cells are observed in either prophase or prometaphase with chromosomes having incorporated BrdU into the early replicating $R$ bands. Once the FPG technique is applied to these preparations, the chromosomes show very sharp G-bands, as can be seen in Figure 1, and karyotyping is made easy since these elongated chromosomes contain a great number of very well contrasted bands. A karyotype of GTGbanded chromosomes obtained after standard culture and trypsinization is depicted in Figure 2. The chromosomes are shorter and the usual less contrasted and more linear banding pattern is observed.

Cell synchronization can be obtained by adding various substances to the culture medium in well calculated amounts sufficient to block the cell cycle, but moderate enough to keep the cells alive. Substances, other than BrdU, that have been used successfully to synchronize horse lymphocytes are methotrexate ${ }^{3}$ and thymidine ${ }^{7}$. It has been observed that these adequate amounts usually block the cell cycle at mid-Sphase where cell synthesis is arrested until rinsing and the addition of appropriate quantities of a synthesis promoting substance. Consequently, cells resume their synthesis in unison, they have been synchronized. Without this procedure, even though their cycle is of approximately the same length, the cells go about their cycle at different times; they do not replicate their DNA, or reach the various mitotic stages all together. Once synchronized, it is easy to choose the appropriate time after mid-S-phase to harvest the cells and fix them at prophase or prometaphase.

The dynamic G-banding pattern is obtained when BrdU is incorporated into the chromosomal DNA during the early-S-phase. Here, this thymidine analogue is taken up into the early replicating $\mathbf{R}$-bands, which will not contract as usual, thus remaining elongated, while other regions of the chromosome, the G-bands, contract normally and are sharply defined. Those regions of DNA that have incorporated BrdU are more sensitive to ultraviolet light and break when exposed to it (photolysis) during the FPG-technique. The contrast is enhanced if the chromosomes have been previously stained with . a fluorescent dye, and Giemsa applied afterwards stains less darkly the broken strands of DNA, thus producing a well contrasted differential staining.

Very often this dynamic pattern manifests itself as symmetrical dots along each chromatid, differing somewhat from the usual linear GTG-banding. Even though both these patterns are not identical, they show enough similarities so that their bands can be compared, establishing the identity of the GB-FPG bands on the longer chromosomes. Finally since GB-FPG and RB-FPG bands are perfectly complementary, the GB-FPG karyotype creates a useful link between the accepted GTG standard and . the newer RB-FPG bands.

Thus, this dynamic G-banding technique that produces many sharply banded prometaphase and prophase chromosomes, can be applied to horse lymphocytes. Indeed, the incorporation of BrdU in chromatin during synthesis constitutes a powerful tool for structural and functional analysis of chromosomes. Furthermore, the Gbands produced by this technique will not only be helpful for cytogenetic diagnosis, but also should play a useful role in the standardization of equine chromosomes.

\section{References}

1. Dutrillaux, B. and E. Viegas-Pequignot. High resolution R-and G-banding on the same preparation. Hum. Genet. 57:93-95. 1981.

2. GAGNE, R. Obtention des bandes " $R$ " par incorporation de BUdr et coloration par le Hoescht 33258 et le Giemsa. Un. Méd. Canada 109:552556.1980.

3. Maciulus, A., T. D. Bunch, J. L. Shupe, and N. C. LEONE. Detailed description and nomenclature of high resolution G-banded horse chromosomes. J. Hered. 75:265-268. 1984.

4. Perry, P. and S. WolfF. New Giemsa method for the differential staining of sister chromatids. Nature 251:156-158. 1974.

5. Romagnano, A., C. L. Richer, and K. J. BetTERIDGE. R-banded chromosomes of the domestic horse obtained, after bromodeoxyuridine incorporation, by the fluorescence-photolysis-Giemsa technique. J. Dairy Sci. 66:suppl. 1:251. 1983.

6. — and - R-banding of horse chromosomes: The fluorescence-photolysis-Giemsa technique after bromodeoxyuridine incorporation. $J$. Hered. 75:269-272. 1984.

7. - and $\longrightarrow$. High resolution R-bands produced in equine chromosomes after incorporation of bromodeoxyuridine. J. Hered. 76:377-378. 1985.

8. SEABRIGHT, M. A rapid banding technique for buman chromosomes. Lancet ii:971-972. 1971. 